\title{
Aspectos clínicos e nutricionais da doença de Gaucher: estudo prospectivo de 13 crianças em um único centro
}

\author{
Clinical and nutritional aspects of Gaucher disease: \\ prospective study of 13 children at a single center
}

\author{
Maria Christina L.A. Oliveira ${ }^{1}$, Benigna M. Oliveira ${ }^{1}$, Elisa Queirós ${ }^{2}$, Marcos B. Viana ${ }^{3}$
}

\section{Resumo}

Objetivo: a doença de Gaucher (DG) é uma doença de depósito lisossomal, de herança autossômica recessiva, causada por mutações do gene $G B A$ (gene da glucocerebrosidase), localizado no cromossomo 1 . O presente estudo foi realizado para identificar as características clínicas, nutricionais, bioquímicas e genéticas dos pacientes com DG acompanhados no serviço de Hematologia Pediátrica do HC-UFMG.

Pacientes e métodos: foram acompanhados prospectivamente 13 pacientes, nos quais o diagnóstico de DG foi confirmado através da determinação da atividade enzimática leucocitária da enzima beta-glucocerebrosidase e/ou genótipo. Foram estudados fatores demográficos, nutricionais e bioquímicos obtidos à admissão. $\mathrm{O}$ estudo genético foi realizado em 7 pacientes. A mediana de seguimento foi de 5,3 anos. Foram calculados o escore $\mathrm{z}$ de peso e estatura na admissão e no final de seguimento. A prevalência padronizada de desnutrição foi calculada pelo método de Mora.

Resultados: a mediana de idade ao diagnóstico foi de 5,8 anos. A manifestação clínica predominante ao diagnóstico foi hepatoesplenomegalia, e todos os pacientes foram classificados como tipo clínico 1. Plaquetopenia ao diagnóstico foi observada em oito crianças, e anemia em seis. A mutação genética mais freqüente foi a N370S. No período de seguimento, foi detectado um óbito por septicemia, após esplenectomia. A prevalência de desnutrição foi de $26 \%$ à admissão, e de $48 \%$ no final do seguimento.

Conclusão: a análise da casuística demonstra que predomina em nosso meio a mutação genética N370S e o tipo clínico 1, caracterizando as formas mais brandas da doença. O uso irregular da medicação não permitiu conclusões clínicas sobre a eficácia da reposição enzimática.

J Pediatr (Rio J) 2002;78(6) 517-22: doença de Gaucher, genótipo, nutrição.

1. Professor Adjunto, Doutor - Dep. Pediatria, Fac. de Medicina, UFMG.

2. Bolsista de iniciação científica - CNPq, Dep. Pediatria, Faculdade de Medicina, UFMG.

3. Professor Titular, Doutor - Dep. Pediatria, Fac. de Medicina, UFMG.

Artigo submetido em 21.05.02, aceito em 14.08.02.

\begin{abstract}
Objective: Gaucher disease is an autosomal recessive lysosomal storage disease, caused by mutation to the $G B A$ gene (glucocerebrosidase gene) located on chromosome 1. The objective of the present study was to identify the clinical, nutritional, biochemical and genetic features of the patients with Gaucher disease treated at the Unit of Pediatric Hematology (Hospital das Clínicas - Universidade Federal de Minas Gerais).
\end{abstract}

Methods: thirteen patients were prospectively followed up. Gaucher disease was confirmed in all patients through the use of an enzyme assay test to measure glucocerebrosidase enzyme activity. Demographic and nutritional data, and biochemical findings obtained on admission, were studied. Genetic testing was performed in 7 patients. The median age of follow-up was 5.3 years. Z scores for weight and height on admission and at the end of observation period were calculated. The standardized prevalence for malnutrition was calculated using the Mora method.

Results: the mean age at diagnosis was 5.8 years. The predominant clinical manifestations at diagnosis were hepatomegaly and splenomegaly, and all patients were classified as clinical type 1 . Low platelet count and anemia were detected at diagnosis in eight and six children, respectively. The most frequent genetic mutation was N370S. One child died during follow-up due to septicemia, after splenectomy. The prevalence of malnutrition was $26 \%$ at admission and $48 \%$ at the end of the observation period.

Conclusion: the analysis of data demonstrates that N370S and clinical type 1 predominate in our Unit, characterizing the milder form of the disease. The irregular enzyme replacement therapy during the study period did not allow valid clinical conclusions on its efficacy.

JPediatr (Rio J) 2002;78(6):517-22: Gaucher disease, genotype, nutrition.

\section{Introdução}

A doença de Gaucher (DG) é a mais comum das doenças de depósito lisossomal ${ }^{1}$, ocorrendo com uma frequiência estimada de 1 em cada 40.000 a 60.000 pessoas na população geral ${ }^{2}$. É mais comum entre as populações judias, sendo 
considerada como a doença genética mais comum entre os judeus Ashkenazi ${ }^{3}$.

Trata-se de uma doença genética autossômica recessiva, causada por mutações do gene GBA (gene da glucocerebrosidase), localizado no braço longo do cromossomo 1. Esse gene codifica a enzima beta-glucocerebrosidase, sendo que mutações genéticas determinam deficiência de sua atividade ${ }^{4}$. Dessa maneira, a DG caracteriza-se pelo acúmulo intralisossomal de glucocerebrosídeo, um glicolipídio derivado de membranas celulares fagocitadas em macrófagos, nos tecidos do sistema reticuloendotelial ${ }^{5,6}$. Essas células carregadas de lipídeos são conhecidas como células de Gaucher.

É uma doença multissistêmica, associada com grande variação em suas manifestações clínicas, na sua gravidade e evolução. Manifesta-se através de anemia, hepatoesplenomegalia e lesões ósseas. Acometimento pulmonar e envolvimento do sistema nervoso central (SNC) são menos freqüentes ${ }^{7}$. É classificada em três tipos, de acordo com a presença, ou ausência de doença primária no SNC: tipo 1 é não neuropática, sendo esta a forma mais comum, responsável por $99 \%$ dos casos; tipo 2 (tipo infantil) com comprometimento grave do SNC na infância; e o tipo $3 \mathrm{com}$ comprometimento leve do SNC, na adolescência ou no adulto jovem ${ }^{8,9}$.

Antes da introdução da terapia de reposição enzimática, no início dos anos 90 , havia somente tratamento paliativo para a morbidade associada à DG, além do aconselhamento genético ${ }^{10}$. Esplenectomia era realizada para aliviar sintomas de compressão abdominal, citopenias e retardo do crescimento. Com a disponibilidade da terapia enzimática, criou-se a perspectiva de melhor qualidade de vida para os pacientes, com a reversão de muitos sintomas ${ }^{11}$.

Com o objetivo de avaliar as características clínicas e bioquímicas dos pacientes com DG acompanhados em nosso serviço, realizamos um estudo prospectivo e observacional, consistindo a amostra estudada de pacientes admitidos de 1986 a 2000.

\section{Pacientes e métodos \\ Pacientes}

Foram acompanhados os pacientes admitidos na unidade de hematologia pediátrica do Hospital das ClínicasUFMG, com sinais sugestivos de doença de depósito (hepatomegalia e/ou esplenomegalia e/ou hiperesplenismo). O diagnóstico de DG foi confirmado em todos os pacientes através da determinação da atividade enzimática leucocitária da enzima beta-glucocerebrosidase e/ou pelo genótipo.

\section{Variáveis estudadas}

As características estudadas tiveram como base os dados obtidos na admissão dos pacientes na unidade, em um protocolo específico, que consistia de dados demográficos, clínicos, nutricionais e laboratoriais.

\section{Avaliação enzimática}

A determinação da atividade enzimática da beta-glucocerebrosidase foi realizada em 12 pacientes, sendo em 11 pela dosagem leucocitária e, em um paciente, pela dosagem em cultura de fibroblastos. Essa análise foi realizada no laboratório de erros inatos do metabolismo (Hospital de Clínicas de Porto Alegre), utilizando-se $10 \mathrm{ml}$ de sangue total em EDTA. Os pacientes que apresentavam atividade de beta-glucocerebrosidase abaixo dos valores de referência (atividade leucocitária entre 10-45 nanomoles/hora/mg de proteína e, em cultura de fibroblastos, entre 350-1.100 nanomoles/hora/mg de proteína) foram considerados portadores de $\mathrm{DG}^{12}$.

\section{Genótipo}

O estudo genético foi realizado em sete pacientes pelo laboratório clínico Lachtermacher (Rio de Janeiro). Foram pesquisadas as quatro mutações mais freqüentes para o gene GBA, N370S, 84GG, L444P e IVS2+1, sendo utilizada a técnica de PCR-RFLP (Polymerase Chain Reaction; Restricted Fragment Length Polymorphism).

\section{Análise estatística}

O banco de dados foi desenvolvido no programa de domínio público Epi-Info, versão $6^{13}$. Trata-se de um estudo descritivo no qual foi utilizada apenas distribuição de frequiência. Para os fatores nutricionais, foi calculado o escore $\mathrm{z}$ de peso e estatura para a idade e sexo à admissão e ao final do seguimento. O cálculo foi obtido do programa EPINUT, incluído no Epi-Info. A prevalência padronizada de desnutrição foi calculada pelo método de Mora ${ }^{14}$.

\section{Aspectos éticos}

O estudo foi aprovado pela Comissão de Ética da UFMG.

\section{Resultados}

Foram acompanhados 13 pacientes (11 famílias), sendo que havia duas famílias nas quais mais de um membro estava afetado (Tabela1). História de consangüinidade esteve presente em duas famílias (três pacientes). Não foi identificada etnia judaica nas famílias estudadas; uma família era de origem libanesa. A idade, ao diagnóstico, das 13 crianças (nove do sexo masculino e quatro do feminino) variou de 1,9 a 10 anos, sendo a mediana de 5,8 anos. Em relação às manifestações clínicas ao diagnóstico, hepatoesplenomegalia, geralmente de grandes proporções, ocorreu em todos os pacientes. As alterações ósseas ocorreram em cinco pacientes, incluindo crises dolorosas, fratura espontânea, desabamento vertebral, osteomielite e necrose asséptica de cabeça de fêmur. Neuropatia e pneumopatia não foram detectadas em nenhum paciente. Ao diagnóstico, nove pacientes apresentavam plaquetopenia (variando de 
18.000 a $148.000 / \mathrm{mm}^{3}$ ) e seis encontravam-se anêmicos $(8,6$ a $9,9 \mathrm{~g} / \mathrm{dl})$; nenhum estava leucopênico. Em relação às provas de função hepática, não foram encontradas alterações bioquímicas. Nove pacientes foram submetidos ao mielograma, sendo que em oito foram encontradas células de Gaucher (Figura 1). De acordo com o tipo clínico da doença, todos os pacientes foram classificados como portadores do tipo 1. A atividade da beta-glucocerebrosidase leucocitária variou de 0,14 a 2,1 nanomoles/hora/mg de proteína. A atividade em fibroblastos foi de 9,3 nanomoles/ hora/mg de proteína no paciente número 12 (Tabela 1).

O estudo genético foi realizado em sete pacientes. No total de 14 cromossomos estudados, a mutação mais freqüente foi a N370S, encontrada em seis cromossomos, seguida da 84GG, que ocorreu em três cromossomos, e pela L444P encontrada em dois cromossomos. Foram identificados 4 pacientes heterozigotos compostos. Em três pacientes, foi identificada heterozigose para apenas uma mutação dentre as pesquisadas.

\section{Curso clínico e tratamento}

A mediana de seguimento dos 13 pacientes foi de 64 meses (3 - 166 meses). Durante o período de acompanha- mento, cinco pacientes foram submetidos à esplenectomia, sendo que em um caso a esplenectomia foi parcial. A mediana de idade na ocasião da esplenectomia foi de 7,2 anos. Destes pacientes, um evoluiu para o óbito, devido à septicemia. Atualmente 10 pacientes (77\%) estão em uso da terapia de reposição enzimática, fornecida regularmente pela Secretária de Saúde do Estado de Minas Gerais. Devido ao alto custo, o fornecimento do medicamento se fez de forma irregular no período estudado, o que não permite a análise adequada da resposta terapêutica.

\section{Dados nutricionais}

A distribuição do escore $\mathrm{z}$ do peso, ao diagnóstico, para as 13 crianças, ficou entre $-2,10 \mathrm{e}+1,98$, sendo a média de $-0,71$. Apenas dois pacientes apresentavam-se com escore $\mathrm{z}$ menor ou igual a $-2,00$. A distribuição do escore $\mathrm{z}$ de peso ao término do seguimento variou entre $-2,21 \mathrm{a}+1,16$, sendo a média de $-0,97$; três crianças ( $23 \%$ ) apresentavam-se, ao final do seguimento, com escore $\mathrm{z}$ menor que $-2,00$.

A distribuição do escore $\mathrm{z}$ da estatura ao diagnóstico variou de $-3,39$ e $+2,64$, sendo a média de $-1,18$; quatro pacientes apresentavam-se com escore $\mathrm{z}$ de estatura menor que $-2,00$. O escore $\mathrm{z}$ para a estatura no final do seguimento ficou entre $-3,98$ e $+1,39$, sendo a média de $-0,91$; três

Tabela 1 - Dados clínicos e laboratoriais em 13 pacientes com doença de Gaucher do tipo 1

\begin{tabular}{|c|c|c|c|c|c|c|c|c|c|c|c|}
\hline \multirow[t]{2}{*}{ Pacientes* } & \multirow{2}{*}{$\begin{array}{c}\text { Idade } \\
\text { diagnóstico } \\
\text { (anos) }\end{array}$} & \multirow[t]{2}{*}{ Sexo } & \multirow[t]{2}{*}{$\begin{array}{c}\text { Genótipo } \\
\text { (mutações) }\end{array}$} & \multirow[t]{2}{*}{$\begin{array}{c}\text { Atividade } \\
\text { enzimática }\end{array}$} & \multirow{2}{*}{$\begin{array}{c}\text { Células de } \\
\text { Gaucher na } \\
\text { medula óssea }\end{array}$} & \multicolumn{2}{|c|}{$\begin{array}{c}\text { Dados } \\
\text { antropométricos } \S\end{array}$} & \multirow{2}{*}{$\begin{array}{l}\text { Alte- } \\
\text { rações } \\
\text { ósseas }\end{array}$} & \multirow{2}{*}{$\begin{array}{c}\text { Pla- } \\
\text { quetas } \\
(x \text { 109/l) }\end{array}$} & \multirow[t]{2}{*}{$\begin{array}{l}\text { Hemo- } \\
\text { globina }\end{array}$} & \multirow{2}{*}{$\begin{array}{l}\text { Segui- } \\
\text { mento } \\
\text { (anos) }\end{array}$} \\
\hline & & & & & & zpeso1/zpeso2 & zestat1/zestat2 & & & & \\
\hline 1 & 3,6 & M & N370S / 84GG & 0,87 & Não realizada & $-1,03 /-0,96$ & $-1,56 / 0,06$ & Sim & 68 & 9,8 & 5,4 \\
\hline $2^{\dagger}$ & 5,8 & $\mathrm{~F}$ & N370S / 84GG & 0,82 & Sim & $1,27 / 1,16$ & $2,64 / 1,39$ & Sim & 18 & 9,3 & 13,8 \\
\hline $3^{\dagger}$ & 6,9 & M & N370S / 84GG & 0,70 & Não realizada & $1,98 /-0,18$ & $-/-0,67$ & Não & 245 & 11,7 & 10,7 \\
\hline 4 & 10,0 & M & N370S / L444P & 0,73 & Sim & $-1,43 /-1,40$ & $-2,28 /-1,41$ & Sim & 148 & 14,5 & 7,5 \\
\hline 5 & 1,9 & M & N370S/outra & 0,49 & Não realizada & $-1,83 /-2,08$ & $-2,49 /-3,98$ & Sim & 208 & 10,5 & 7,3 \\
\hline 6 & 7,0 & $\mathrm{~F}$ & N370S/outra & Não & Sim & $-0,48 /-2,21$ & $-1,15 /-0,91$ & Sim & 216 & 9,5 & 9,3 \\
\hline 7 & 2,9 & $\mathrm{M}$ & L444P/outra & 0,32 & Não realizada & $0,54 / 0,28$ & $-/-1,04$ & Não & 123 & 11,0 & 7,1 \\
\hline $8^{\dagger+}$ & 4,2 & M & Não realizado & 0,80 & Sim & $-1,29 /-0,90$ & $0,53 / 0,40$ & Não & 114 & 11,3 & 2,4 \\
\hline $9^{\dagger \neq}$ & 3,7 & M & Não realizado & 0,14 & Sim & $-0,57 / 0,26$ & $-0,65 / 0,01$ & Não & 62 & 9,1 & 0,7 \\
\hline $10^{\ddagger}$ & 6,4 & $\mathrm{~F}$ & Não realizado & 1,74 & Não encontrada & $-0,69 /-0,50$ & $-0,68 / 0,94$ & Não & 20 & 11,2 & 5,4 \\
\hline 11 & 5,9 & M & Não realizado & 2,10 & Sim & $-2,06 /-2,07$ & $-1,87 /-2,11$ & Não & 56 & 9,9 & 0,3 \\
\hline 12 & 5,8 & M & Não realizado & $9,3 \|$ & Sim & $-2,10 /-2,07$ & $-3,39 /-2,55$ & Não & 173 & 8,6 & 3,5 \\
\hline 13 & 5,8 & $\mathrm{~F}$ & Não realizado & 1,10 & Sim & $-1,55 /-1,99$ & $-2,06 /-1,96$ & Não & 78 & 11,1 & 4,2 \\
\hline Mediana & 5,8 & 5,3 & & & & & & & & & \\
\hline
\end{tabular}

* Todos os pacientes apresentavam hepatosplenomegalia; nenhum apresentava alteração neurológica.

† Os pacientes 2 e 3, bem como 8 e 9, são irmãos.

‡ Filhos de pais consangüíneos.

$\S$ z peso1: escore $z$ do peso em relação ao sexo e idade à admissão; z peso2: escore z ao final do seguimento. $z$ estat1: escore $z$ da estatura em relação ao sexo e idade à admissão; $z$ estat2: escore $z$ ao final do seguimento.

\| Análise da atividade enzimática em cultura de fibroblastos obtida por biópsia de pele. 


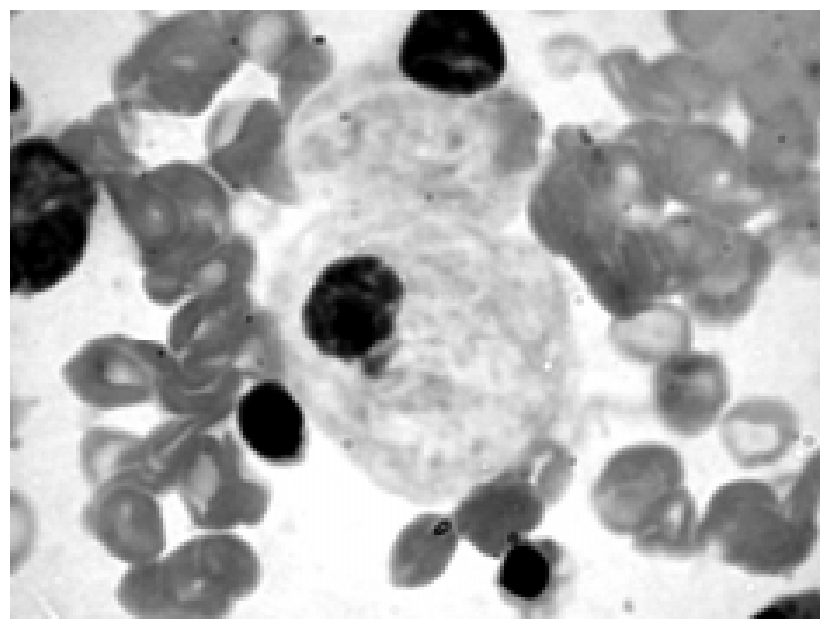

Figura 1 - Fotomicrografia de célula de Gaucher na medula óssea. May-Grünwald-Giemsa, aumento de 1.000X

pacientes $(23 \%)$ apresentavam-se com escore $\mathrm{z}$ de estatura menor que $-2,00$.

A prevalência padronizada de desnutrição, calculada pelo método de Mora, demonstrou um percentual de $26 \%$ de desnutridos, considerando-se o peso de admissão. Ao final do seguimento, a prevalência padronizada de desnutrição, considerando-se o peso final, foi de $48 \%$.

\section{Discussão}

O conhecimento do curso clínico da DG torna-se particularmente importante em relação às decisões quanto à melhor abordagem para os pacientes afetados pela doença. Dois aspectos relevantes são fundamentais na compreensão das questões da abordagem atual da DG. Primeiro, a marcante heterogeneidade da doença em relação à apresentação, ao curso clínico e ao prognóstico. O segundo aspecto é o desenvolvimento de novas terapêuticas de custo elevado, como a terapia de reposição enzimática e o transplante de medula óssea.

A estimativa precisa da freqüência da DGé difícil de ser obtida, sendo que poucas informações são disponíveis sobre populações não judias. Entretanto, registros da DG, em vários países, estimam que a doença tem uma frequiência que varia de 1 para 40.000 a 1 para 200.000 na população geral não-judia. Assim, acredita-se que aproximadamente 30.000 pessoas são portadoras de DG em todo o mundo ${ }^{9}$. A DG nãoé comum em nosso meio. O mais abrangente estudo é originado de um centro de referência para detecção de erros inatos do metabolismo ${ }^{15}$. Nesse estudo, a DG foi responsável por $3,6 \%$ de uma população de 10.000 crianças com sinais e sintomas sugestivos de erros inatos do metabolismo.
A partir de 1995, foi criado o International Collaborative Gaucher Group (ICGG), o maior banco de dados do mundo de pacientes portadores de DG. Foi estabelecido, assim, o primeiro passo na melhor compreensão da história natural da doença, o que permitirá o desenvolvimento de medidas de monitorização e tratamento adequados ${ }^{16}$.

Neste registro, foram compilados dados de 1.698 pacientes de aproximadamente 38 países. O Brasil participa com menos de $1 \%$ da amostra, referente a um único centro. Assim, comparar dados de registros multicêntricos com dados de casuística de um único centro, com menor número de pacientes, pode ser inapropriado. Mas não se pode deixar de comentar que alguns dados, como a freqüência das mutações entre os pacientes observados em nosso serviço, estão próximos daqueles detectados nas séries de outros centros especializados.

O curso clínico da DG pode variar consideravelmente, mesmo dentro de uma mesma família, compartilhando o mesmo genótipo ${ }^{17}$. Essa heterogeneidade observada na manifestação clínica inicial, idade ao diagnóstico e posterior progressão da doença, tem sido atribuída, pelo menos em parte, ao tipo de mutação observada. Desse modo, tem sido proposto o conceito de mutações leves e graves, para explicar a heterogeneidade clínica da doença. A correlação do genótipo com o fenótipo permanece obscura. Entretanto, pacientes homozigotos para a mutação N370S são diagnosticados mais tardiamente e apresentam a doença do tipo 1. Já os pacientes que apresentam um fenótipo mais grave, com comprometimento neurológico, em geral são homozigotos para a mutação L444P, e diagnosticados mais precocemente ${ }^{16}$. Além disso, fatores ambientais desconhecidos parecem ser críticos para a expressão da doença ${ }^{9}$.

Desde que o gene da DG foi identificado, aproximadamente 109 mutações foram relatadas ${ }^{18}$. Entre os judeus Ashkenazi, quatro mutações são responsáveis por $90 \%$ dos casos, N370S, 84GG, L444P, e IVS2+1. Na população não-judia, a doença apresenta baixa prevalência, e as mutações mais comumente identificadas são as L444P, N370S, D409H, R463C, e IVS2 $+1^{19}$. No nosso estudo, nenhum dos sete pacientes analisados era homozigoto para as mutações pesquisadas. Dos 14 alelos (7 pacientes) analisados, a mutação mais freqüente foi a N370S (42\%), seguida pela mutação 84GG (21\%) e pela L444P (14\%). Esses dados são semelhantes aos encontrados em uma série no Canadá, na qual, após a análise de 6 pacientes, as mutações mais freqüentemente encontradas foram a N370S $(42 \%)$ e a L444P $(58 \%)^{20}$. No Gaucher Registry, dentre os 1.528 cromossomos analisados, a mutação mais comum identificada foi a N 370S (53\%), seguida pela L444P (18\%), a 84GG (7\%), e IVS2+1 (2\%) ${ }^{16}$.

A média de idade ao diagnóstico para os nossos pacientes foi de 5,8 anos, sendo bem mais jovem que a relatada em outras séries. Entre os pacientes cadastrados no ICGG, a média de idade ao diagnóstico foi de 17,4 anos, variando do nascimento a 81 anos. Contudo, no registro internacional, 
mais da metade dos pacientes foi diagnosticada antes dos dez anos de idade. Essa amplitude pode refletir a heterogeneidade dos métodos de diagnóstico utilizados nos diversos países que participam do registro.

Em relação às manifestações clínicas, como anemia e plaquetopenia, sua freqüência foi relevante em nossa série, acometendo $43 \%$ e $64 \%$ dos pacientes, respectivamente. Esses dados são comparáveis aos encontrados no ICGG, no qual a freqüência alcança até $2 / 3$ dos pacientes ${ }^{16}$.

O prognóstico dos pacientes com DG tem melhorado nos últimos anos. Isto tem sido atribuído ao diagnóstico mais precoce e à terapia de suporte instituída. O prognóstico depende das complicações hematológicas, viscerais, ósseas ou infecciosas. O estado nutricional ainda não tem sido abordado na literatura. Em nossa série, foi demonstrada uma piora do parâmetro nutricional, avaliado ao longo do seguimento. Esse fato pode ser secundário à falta de tratamento regular, ou inerente à própria doença, sendo necessários estudos com maior amostragem, para que sejam obtidas evidências mais conclusivas.

Embora as anormalidades hematológicas e viscerais possam ser graves e potencialmente fatais, é o acometimento ósseo, em geral, o principal determinante da morbidade e incapacidade a longo prazo ${ }^{21}$. Há um amplo espectro de complicações esqueléticas relacionadas à DG, variando de osteopenia assintomática à osteonecrose com degeneração articular secundária ${ }^{22}$. Apesar de muitas lesões ósseas responderem ao tratamento de reposição enzimática, a osteonecrose, a osteoesclerose e a compressão vertebral parecem ser irreversíveis ${ }^{23}$. Em nossa série, não foi possível a avaliação sistemática do sistema esquelético, embora fratura espontânea e desabamento vertebral tenham sido observados.

Septicemia é uma causa proeminente de morte na DG. Pode ser consequiência da esplenectomia, de um defeito qualitativo e reversível na migração de neutrófilos e de outros fatores predisponentes à doença séptica ${ }^{24}$. Observase, também, déficit na quimiotaxia dos neutrófilos, aumentando o risco de infecção ${ }^{25}$. A atividade enzimática, entretanto, não é um fator prognóstico da gravidade da doença ${ }^{26}$. Em nossa série, no período de seguimento, houve um óbito secundário à septicemia, pós-esplenectomia.

A terapia de reposição enzimática para DG tem provado ser segura e efetiva, após quase uma década de uso em mais de 2.500 pacientes em todo o mundo ${ }^{27}$. Indicadores de boa resposta ao tratamento incluem aumento no nível de hemoglobina e da contagem de plaquetas e diminuição na hepatoesplenomegalia. Entretanto, a melhora dos sintomas ósseos pode levar anos para se tornar evidente ${ }^{28}$. Aqueles portadores de doença de Gaucher tipo 1 apresentam boa resposta ao tratamento enzimático. Existem poucos dados na literatura sobre o tratamento da doença de Gaucher tipo 2 e $3^{29}$. Ainda não está claro como a terapia de substituição enzimática seria efetiva na situação de comprometimento do SNC, uma vez que a enzima penetra mal na barreira hematoencefálica. A redução do depósito da substância no organismo poderia, entretanto, ajudar a prevenir o acúmulo no cérebro ${ }^{30}$. Dados recentes do International Collaborative Group relatam que a freqüência da crise de infarto ósseo é reduzida em $90 \%$ a $95 \%$ dos pacientes recebendo terapia de substituição enzimática.

Todas as evidências sugerem que a terapia enzimática tem o potencial de prolongar a vida dos pacientes com DG, diminuindo as complicações que causam risco de vida ${ }^{31}$. Evidências de que a terapia enzimática também melhora o defeito qualitativo na quimiotaxia dos neutrófilos e corrige as alterações de coagulação que ocorrem na DG tipo I proporcionam motivos adicionais de otimismo ${ }^{24}$. A casuística apresentada no presente estudo não permite conclusões sobre a eficácia da terapêutica, pois, conforme já exposto, o uso da reposição enzimática foi irregular no período de estudo, o que, com certeza, compromete a resposta clínica e a qualidade de vida dos pacientes.

Quanto ao transplante de medula óssea, não é recomendado para os pacientes com DG tipo 1. Para aqueles pacientes com o tipo 3, após serem submetidos ao transplante, apresentam remissão dos sintomas, e existem indícios de que a progressão da doença neurológica possa ser interrompida $^{32}$.

Somente a partir de um acompanhamento clínico e laboratorial, com a participação de equipe multidisciplinar e grupos cooperativos multicêntricos, será possível avaliar a contribuição do genótipo, de fatores ambientais e étnicos e de outras variáveis na história natural da doença, bem como a eventual interferência da terapêutica específica na evolução de determinados sinais e sintomas.

\section{Agradecimentos}

Os autores agradecem ao Dr. Roberto Giugliani, coordenador do Laboratório de Erros Inatos do Metabolismo (Hospital de Clínicas de Porto Alegre) e à sua equipe pela determinação da atividade enzimática leucocitária da betaglucocerebrosidase, e ao Laboratório Clínico Lachtermacher e à Genzyme do Brasil, pela realização do estudo genético. A aluna E. Queirós recebeu bolsa de iniciação científica do CNPq.

\section{Referências bibliográficas}

1. Beutler E. Commentary: the natural history of Gaucher disease. Blood Cells Mol Dis 1998;24:82.

2. Grabowski GA, Saal HM, Wenstrup RJ, Barton NW. Gaucher disease: a prototype for molecular medicine. Crit Rev Oncol Hematol 1996;23:25-55. 
3. Charrow J, Esplin JA, Gribble TJ, Kaplan P, Kolodny EH, Pastores GM, et al. Gaucher disease: recommendations on diagnosis, evaluation, and monitoring. Arch Intern Med 1998;158:1754-60.

4. Sorge J, West C, Westwood B, Beutler E. Molecular cloning and nucleotide sequence of human glucocerebrosidase cDNA. Proc Natl Acad Sci U SA 1985;82:7289-93.

5. Meikle PJ, Hopwood JJ, Clague AE, Carey WF. Prevalence of lysosomal storage disorders. JAMA 1999;281:249-54.

6. Parkin JL, Brunning RD. Pathology of the Gaucher cell. Prog Clin Biol Res 1982;95:151-75.

7. Barranger JA, Tomich J, Weiler S, Sakallah S, Sansieri C, Mifflin T, et al. Molecular biology of glucocerebrosidase and the treatment of Gaucher disease. Cytokines Mol Ther 1995;1:149-63.

8. Beutler E. Gaucher's disease. N Engl J Med 1991;325:1354-60.

9. Cox TM, Schofield JP. Gaucher's disease: clinical features and natural history. Baillieres Clin Haematol 1997;10:657-89.

10. Barton NW, Brady RO, Dambrosia JM, Di Bisceglie AM, Doppelt SH, Hill SC, et al. Replacement therapy for inherited enzyme deficiency - macrophage-targeted glucocerebrosidase for Gaucher's disease. N Engl J Med 1991; 324:1464-70.

11. Mistry PK, Abrahamov A. A practical approach to diagnosis and management of Gaucher's disease. Baillieres Clin Haematol 1997;10:817-38.

12. Harzer K. Enzymic diagnosis in 27 cases with Gaucher's disease. Clin Chim Acta 1980; 106:9-15.

13. Dean AG, Dean JA, Coulombier D. Epi Info, Version 6: a word processing, database, and statistics program for epidemiology on microcomputers. Centers for Disease Control and Prevention, Atlanta, Georgia, U.S.A., 1994.

14. Mora JO. A new method for estimating a standardized prevalence of child malnutrition from anthropometric indicators. Bull World Health Organ 1989;67:133-42.

15. Coelho JC, Wajner M, Burin MG, Vargas CR, Giugliani R. Selective screening of 10,000 high-risk Brazilian patients for the detection of inborn errors of metabolism. Eur J Pediatr 1997; 156:650-4.

16. Charrow J, Andersson HC, Kaplan P, Kolodny E H, Mistry P, Pastores G, et al. The Gaucher registry: demographics and disease characteristics of 1698 patients with Gaucher disease. Arch Intern Med 2000;160:2835-43.

17. Maaswinkel-Mooij P, Hollak C, van Eysden-Plaisier M, Prins M, Aerts H, Poll R. The natural course of Gaucher disease in The Netherlands: implications for monitoring of disease manifestations. J Inherit Metab Dis 2000;23:77-82.

18. Beutler E, Gelbart T. Hematologically important mutations: Gaucher disease. Blood Cells Mol Dis 1998;24:2-8.

19. Horowitz M, Zimran A. Mutations causing Gaucher disease. Hum Mutat 1994;3:1-11.

20. Devost NC, Choy FY. Mutation analysis of Gaucher disease using dot-blood samples on FTA filter paper. Am J Med Genet 2000;94:417-20.
21. Hermann G, Goldblatt J, Levy RN, Goldsmith SJ, Desnick RJ, Grabowski GA. Gaucher's disease type 1: assessment of bone involvement by CT and scintigraphy. AJR Am J Roentgenol 1986;147:943-8.

22. Pastores GM, Patel MJ, Firooznia H. Bone and joint complications related to Gaucher disease. Curr Rheumatol Rep 2000;2:175-80.

23. Pastores GM, Hermann G, Norton K, Desnick RJ. Resolution of a proximal humeral defect in type-1 Gaucher disease by enzyme replacement therapy. Pediatr Radiol 1995;25:486-7.

24. Zimran A, Abrahamov A, Aker M, Matzner Y. Correction of neutrophil chemotaxis defect in patients with Gaucher disease by low-dose enzyme replacement therapy. Am J Hematol 1993; 43:69-71.

25. Zimran A, Gelbart T, Westwood B, Grabowski GA, Beutler E. High frequency of the Gaucher disease mutation at nucleotide 1226 among Ashkenazi Jews. Am J Hum Genet 1991;49:855-9.

26. Grabowski GA. Current issues in enzyme therapy for Gaucher disease. Drugs 1996;52:159-67.

27. Elstein D, Abrahamov A, Hadas-Halpern I, Zimran A. Recommendations for diagnosis, evaluation, and monitoring of patients with Gaucher disease. Arch Intern Med 1999;159:1254-5.

28. Rosenthal DI, Doppelt SH, Mankin HJ, Dambrosia JM, Xavier RJ, McKusick KA, et al. Enzyme replacement therapy for Gaucher disease: skeletal responses to macrophage-targeted glucocerebrosidase. Pediatrics 1995;96:629-37.

29. Bove KE, Daugherty C, Grabowski GA. Pathological findings in Gaucher disease type 2 patients following enzyme therapy. Hum Pathol 1995;26:1040-5.

30. Rice EO, Mifflin TE, Sakallah S, Lee RE, Sansieri CA, Barranger JA. Gaucher disease: studies of phenotype, molecular diagnosis and treatment. Clin Genet 1996;49:111-8.

31. Beutler E, Demina A, Laubscher K, Garver P, Gelbart T, Balicki $\mathrm{D}$, et al. The clinical course of treated and untreated Gaucher disease. A study of 45 patients. Blood Cells Mol Dis 1995;21: 86-108.

32. Erikson A, Groth CG, Mansson JE, Percy A, Ringden O, Svennerholm L. Clinical and biochemical outcome of marrow transplantation for Gaucher disease of the Norrbottnian type. Acta Paediatr Scand 1990;79:680-5.

Endereço para correspondência:

Dra. Maria Christina Lopes A. Oliveira

Rua Patagônia, 515/701 - Sion

CEP 30320-080 - Belo Horizonte, MG

Fone: (31) 3285.1056 - Fax: (31) 3222.3584

E-mail:eduolive@medicina.ufmg.br 